\title{
THE PRIMARY VENTRAL ROOTS AND SOMATIC MOTOR COLUMN OF AMBLYSTOMA
}

\author{
G. E. COGHLLL \\ Denison University, Granmille, Ohio \\ TWENTY-EIGHT FIGURES
}

Preliminary to the publication of studies upon the development of the nervous system in relation to the development of behavior in embryos of Amblystoma this paper is intended to record and discuss briefly certain observations which, beyond their relation to the general problem of correlation of growth and function, have particular bearing upon the morphology of the nervous system. These observations have to do with the nature of the primary somatic motor column of the spinal cord and brain and the relation of the primary ventral roots to the neurones of this column. The general conception, growing out of the study of the definitive ventral horn cell, seems to have been that the neurones which form the ventral roots are a distinct type. The neurones, however, which establish the earliest contact with the cells of the myotome are found in Amblystoma to be at the same time the neurones of the motor tract in the central nervous system. The primary ventral root fiber is a collateral of a tract cell. It is this discovery with which this communication is concerned.

The species used chiefly in these studies is A. punctatum. Occasionally A. opacum has been introduced but my observations do not suggest that there is any difference between the two species on the point under consideration. The specimens were selected according to the physiological standards which an earlier paper ${ }^{1}$ describes in detail. Thest standards are based on the ability of the embryo to execute somatic movements in

Jour. Comp. Neur., vol. 19, no. 1. 
response to tactile stimulation. In order to obtain specimens that are approaching the stage of earliest reaction to touch my method has been to apply the current from an inductorium gauged so as to stimulate a slightly older individual without injury. This test differentiates the embryos that are very near to the stage in which response to tactile stimulation becomes possible, for the somatic muscles can be stimulated by electricity in this manner for a brief period before they can be stimulated through the tactile receptors. Embryos in this phase of development represent the earliest stage taken account of in this study. Following this, in the natural order of development, are specimens of the early flexure stage (which contract only the most rostral myotomes), specimens of the 'coil-reaction' stage (which bend the trunk into a tight coil), specimens in the 'S-reaction' stage (which perform a compound flexure or sinuous movement) and specimens of the early swimming stage. The latter specimens are approximately $7 \mathrm{~mm}$. long. Drawings and descriptions of the root fibers of all these stages and remarks upon the methods of fixation and preparation are given in connection with the explanation of the figures. The scope of the paper is in no sense intended to cover or take account of the literature upon the subject. This part of the work must be deferred till the publication of the general results of my studies upon correlation of growth and function.

\section{CONDITIONS FOUND IN PARTICULAR CASES}

\section{Embryos reacting to electricity but not to touch}

The conditions of the root fibers as they occur in the youngest embryos of my collection, that is to say, in embryos which react to electrical but not to tactile stimulation, are illustrated in figures 1 and 2. The plane of section in figure 1 is approximately frontal, but tipped slightly ventro-laterad on the side figured. The most ventral portion of the central canal appears in the section, although the figure reaches only about half way from the periphery of the cord to the canal. The more mesial; elongated nuclei of the figure represent the nuclear characteristics 
of the more central portion of the section. Near the periphery of the cord are nuclei of very different form. They are large and approximately spherical. The indistinctly differentiated perikarya to which these nuclei belong $(V C)$ are crowded with yolk spherules, some of which occupy indentations in the nuclei. In fact the nuclei seem to partially surround such spherules in an ameboid fashion. Among these peripheral cells of figure 1 are at least two $(V C)$ which clearly belong to the motor column. The more rostral one has a distinctly differentiated descending process $(D P)$ which runs longitudinally immediately within the external limiting membrane of the cord. There is also a suggestion of an ascending process. The absence of a clearly differentiated ascending process in the case of this neurone is explained by the appearance of the more caudal neurone $(V C)$ which lacks its nucleus and descending process. Obviously the plane of section is such that a section which passes through the nucleus of a tract cell includes the descending but not the ascending process; while a section which passes a little ventrad of the nucleus, as the section does in this case, strikes through the ascending process. Accordingly the section next dorsad of this shows a typical tract cell nucleus in the position that corresponds with this neurone and not less than three others of the same class distributed more caudally in a series. The more caudal neurone of this figure, then, belongs also to the motor column, and from its ascending process arises a branch which passes through the external limiting membrane as a root fiber to the third myotome. In this section this root fiber reaches the anlage of the spinal ganglion, among the cells of which it can be recognized in the adjacent section.

Since the first post-otic myotome ordinarily has no motor root (a root has been found in at least one instance), the root represented in figure $1(V F)$ is the second motor root and is associated with the anlage of the first spinal ganglion. The root to the second myotome in this specimen can also be identified. While the plane of section through it does not permit positive conclusions regarding its mode of origin the root fiber certainly either 
passes out of the tract from caudad as an axone or arises as a collateral from the ascending process of a tract cell.

Traced caudad in this embryo the fibers of the motor column can be identified as far as the fourteenth myotome. Beyond this a single fiber may be seen in occasional sections as far as the seventeenth myotome. Ventral root fibers can be recognized as far caudad as the thirteenth myotome, that is, there are at least twelve pairs of ventral roots in this specimen.

The root fiber indicated in figure $2(V F)$ goes to the fifth myotome of an embryo of the same physiological age as that from which figure 1 was taken. Although the cell body of the neurone from which the root fiber arises does not appear in this section, the tract fiber has the appearance of a descending process $(D P)$. The slight outward bend of the fiber at the point of origin of the collateral which passes into the nerve root is characteristic of this early stage of development.

Slightly rostrad of the root fiber in this section is a characteristic nucleus of the motor column and its perikaryon can be indistinctly seen $(V C)$. The peripheral end of this cell spreads out against the external limiting membrane and extends rostrad and caudad in ascending and descending processes.

Root fibers occur in this embryo as far caudad as the eighth myotome, and uncertain suggestions of roots appear at the levels of the ninth and tenth myotomes. The fibers of the latero-ventral tract may be traced caudad as far as the twelfth myotome.

\section{Embryos of the early flexure stage}

Conditions found in embryos very soon after they first respond to tactile stimulation are represented in figures 3 to 9 , inclusive.

The section from which figure 3 is taken is in an approximately frontal plane, tipped slightly ventro-caudad and slightly dorsolaterad on the side figured, so as to leave a point of the lateroventral portion of the spinal cord between the notochord and the eighth myotome. The descending process of a neurone $(D P)$, the nucleus of which is not included in this section, branches into two divisions close to the external limiting membrane. One 
division, apparently the larger in this case, passes out of the cord as a root fiber $(V F)$ and reaches almost to the anlage of the spinal ganglion $(S G)$. The other division continues caudad in the latero-ventral tract. Descending processes of other cells enter this root, as shown in the figure, without giving any evidence of bifurcation beneath the limiting membrane, but there is nothing in the preparation that would render such a branching improbable. In fact, slightly caudad of the root are clearly differentiated fibers of the tract which may well be the descending processes of these same neurones.

Figure 4 is drawn from the opposite side of the same embryo and the section is therefore tipped slightly latero-ventrad. It shows a root fiber $(V F)$ arising clearly as a collateral of a tract fiber and passing through the anlage of the spinal ganglion to the muscle cell $(M C)$. Its ending upon this muscle cell can not be regarded as clearly demonstrable in this section but the fiber expands slightly upon the surface of the cell and apparently ends there. Just caudad of the region of this root collateral is the basal portion of another fiber which almost certainly has the same relation to the neurone of the tract. The origin of these root fibers from the tract opposite the middle of the myotome and their application to the muscle cell directly opposite a large nucleus against which impinges a conspicuous mass of pigment $(P)$ is characteristic of these early neuro-muscular relations. The outward thrust of the axone of the tract at the point of origin of the root collateral is also characteristic, as shown in figure 4 .

The form of the neurones of the motor column is illustrated in figure $5(V C)$. The neurone of the figure occurs at the level of the thirteenth myotome, some muscle cells of which $(M C)$ are shown in the figure. The position and general relations of this neurone in the cord are-shown in figure $6(V C)$, from which the plane of section of figure 5 can be readily interpreted. Although the portion of the cell visible in this section is only about half the length of the adjacent myotome, the entire cell probably exceeds the myotome in length. Another neurone of this column, located at the level of the twenty-first and twenty-second myotomes (fig. 8, $V C, M$ ) is shown in figure 7, and its general rela- 
tions in the cord are shown in figure 8. In this case both the descending and ascending processes can be readily followed for some distance, and the ascending process, presumably the dendrite, is branched. There appears, also, to be a small branch off of the descending process $(D P)$ near its base, while the main process ends abruptly and in such relations as to indicate that it actually extends a considerable distance beyond the limits of this section. The tangential section of the nucleus of this neurone, as shown in the figure, is in keeping with the form of the tract cell as demonstrated in other planes of section, which show the neurones with their perikarya shunted off to one side of the main fibrillar axis. The distribution of the yolk spherules in accordance with the fibrillar structure around the nucleus, as shown in this figure, is typical.

There is no evidence of ventral roots in this embryo at the level of the cell of figure 7 ; and the general appearance of the myotomes of that level as shown in figure 8 would not lead one to expect ventral roots at this level. However, a study of the two adjacent myotomes throughout their entire extent in the serial sections reveals a few cells in their most ventro-mesial portion that might have muscular function. Comparative studies of embryos, on the other hand, lead me to believe that at this stage the ventral root system has not extended so far caudad, and that this tract cell, therefore, lies considerably caudad of any ventral root.

Another case of the origin of a ventral root fiber as a collateral from a tract neurone in an embryo of this age is shown in figure 9. Here the plane of section is in general vertical, but the embryo is twisted in such a way that at the level of this root the section is very nearly in the frontal plane, being tipped considerably latero-ventrad on the side figured. A careful study of this root fiber $(V F)$ by varying the focal plane gives one the impression that the fiber from which the collateral arises is a descending process of a tract neurone, and the angles subtended by the collateral and the axone corroborate this interpretation. Here again is seen the characteristic outward thrust of the axone at the point of origin of the root collateral. 


\section{Embryos of the 'coil-reaction' stage}

The origin of root fibers at various levels in embryos of the 'coil-reaction' stage is shown in figures 10 to 15 , inclusive.

The root of figure 10 goes to the seventh myotome. The figure is drawn from an approximately frontal section, tipped slightly dorso-laterad on the side figured. There are several fibers in the root, towards the base of which the fibers of the tract arch outward. From one of these a root fiber $(V F)$ clearly arises as a collateral; in the case of another, not clearly shown in the figure, the same mode of origin is practically certain. The opposite root to the seventh myotome is drawn in figures 12 and $13(V F)$. In this case the root process of the cell extends directly outward through the external limiting membrane, immediately beneath which ascending and descending processes are given off into the tract. Figure 12 shows the focal plane in which the connection of this neurone with the nerve root is clearest, while figure 13 shows the appearance in the focal plane which demonstrates the relation of the neurone to the ventro-lateral tract.

The root to the eleventh myotome of the same specimen is shown in figure 11, where the collateral $(V F)$ arises from the descending process of a neurone of the motor column. While the cell body can not be distinctly made out in this section the general form of the process leaves no doubt as to its nature. Again, in this section, the arching outward of the axone at the origin of the root collateral is conspicuous.

Far caudad of demonstrable ventral roots in this specimen may be recognized the longitudinally oriented neurones of the motor column. One of these neurones, located at the level of the thirtythird myotome, is drawn in figure $14(V C)$. This neurone lies in the extreme ventro-lateral region of the spinal cord. Its descending process $(D P)$ is more clearly seen in the section than is its ascending process, though this inequality may be due to the plane of section. The yolk spherules in the basal portion of the descending process conform to the polarization of the cell. At this level and in this plane of section the medullary tube is constituted of a single layer of short epithelial cells, excepting as the 
neurones of the ventral column and the neurones of the dorsal column, the giant ganglion cells, are differentiated in the most peripheral part.

The structure of the myotome opposite this root is drawn in figure 15. Careful examination of this myotome and others of the immediate region gives no evidence of their having differentiated muscle cells in any part. This fact, together with the fact that in specimens that are physiologically much more advanced in development than that from which this figure was taken, root fibers can be demonstrated only as far caudad as the twenty-seventh myotome, leaves no doubt that, in this level of the cord, the ventro-lateral tract neurones become differentiated and oriented considerably in advance of the development of ventral roots.

\section{Embryos of the 'S-reaction' stage}

A group of neurones of the motor column is shown in figure 16 . These neurones are found at the level of the twenty-second myotome of an embryo of the 'S-reaction' stage, that is, an embryo that can perform the sinuous, double flexure, but can not perform this movement in series so as to effect locomotion. The plane of section here is approximately frontal, but tipped slightly dorsolaterad on the side figured and also slightly dorso-rostrad. The figure, accordingly, shows the ascending processes $(A P)$ to better advantage than it does the descending processes $(D P)$, though considerable portions of the latter are perceptible in one or two of the neurones. It is possible here to demonstrate the ascending process $(A P)$, presumably the dendrite, of one of these tract neurones for a distance of one-half the length of the myotome. The descending process, my general observations lead me to believe, is ordinarily considerably longer than the ascending process. If this is true, the entire length of one of the tract neurones at this level and in this stage of development must be considerably greater than the length of the myotome.

The relation of the neurones of the motor column to those of other parts of the cord at this age is shown in figure 17. This figure is taken from a section approximately parallel to the sagit- 
tal plane. In the ventral part is seen the ventro-lateral tract $(V T)$ into which the broad processes of the neurones of the motor column $(V C)$ project. In the dorsal region appear the perikarya of the giant ganglion cells which form the dorsal sensory column. The nuclei in this figure are all drawn with the aid of the camera lucida. A general idea, therefore, can be formed from this drawing of the relative size and similarity in arrangement of these two types of neurones. Here the nuclei of the motor column are distinctly smaller than those of the giant ganglion cells, and my observations lead me to believe that they are generally so. It is noteworthy also that while there is a sharp differentiation of the giant ganglion cells from all others around them, there is more or less of a gradual transition from the typical, large, round nucleus of the motor column and the elongated nuclei of the ependyma. A corresponding gradation in nuclei is also seen in certain staining reactions. Such difference in differentiation is obviously correlated with the difference there is between these motor and sensory cells in the further development of the animal. The giant ganglion cells are transitory and do not become permanently worked into the definite organization of the spinal cord, while the motor eells are part of a developing system which elaborates with the development of the animal. The motor system, therefore, is here a developing system whereas the dorsal ganglion cell system has reached its full development so far as cell differentiation is concerned.

\section{Embryos of the early swimming stage}

The general relation of the motor and sensory neurones of the cord in an older specimen is shown in figures 18 and 19. Figure 19 is drawn through the cord at the level of the ninth myotome, while figure 18 is taken from the level of the eighteenth myotome. The ascending process of the giant ganglion cells $(D C)$ may be seen here projecting slightly ventrad toward the dorso-lateral tract $(D T)$. The neurones of the motor column $(V C)$ lie mesially or slightly dorsally of the ventro-lateral tract $(V T)$. Their peripheral ends project into the tract and bifurcate into ascend- 
ing and descending processes, relations that are clearly demonstrated in other planes of section. For instance, in figure 20, taken from an embryo of the early swimming stage, is drawn a neurone of the motor column at the level of the thirtieth myotome. The plane of section here is approximately frontal, but tipped slightly ventro-caudad and dorso-laterad on the side figured. The entire section of the thirtieth myotome is also shown in the figure, and the section through the skin as well. The broad body of the cell, with its characteristic enclosure of yolk spherules, projects laterad against the external limiting membrane and extends caudad in a slender descending process $(D P)$. The basal portion of its ascending process is also perceptible. The position of this neurone of the motor column is considerably caudad of any demonstrable nerve roots.

At the level of the twelfth myotome of an animal of the same degree of development the mode of origin of both the ventral roots is perfectly clear and the conditions are reproduced in figures 24 to 26 , inclusive. In figure 24 a number of fibers sweep from cephalad out of the latero-ventral tract $(V T)$ into the ventral root which passes directly into the anlage of the spinal ganglion $(S G)$. One of these fibers $(D P)$, isolated from the others, shows the branched condition and the collateral $(V F)$ to the root. The characteristic loop made by the fibers of the tract at the origin of the root is better shown in the root of the opposite side, as drawn in figure 25 . In the section of figure 24 the caudal arm of the loop is cut away excepting for a few scattering fibers. In figure 25 both the rostral and caudal arms of the loop are clearly seen, and one of the fibers of the loop shows the branched condition distinctly. This fiber is drawn alone in figure 26 , where the root fiber $(V F)$ is seen as a collateral of a fiber which continues caudad $(D P)$ in the latero-ventral tract. A neurofibril is shown going into each of these divisions of the fiber. In figure 24 there is apparently a branching of a single neurofibril with one branch going into each of the processes, but in this; and in other similar cases, two fibrils may be in very close apposition with one another so that their separation in the given plane of section appears like branching. Such appearance occurs also in figures 21,22 and 27. 
Figure 27 is taken from the level of the tenth myotome of the same specimen as that of figure 24. Here, again, the loop of the tract fibers is perceptible, particularly in the case of the fiber from which the collateral $(V F)$ arises. This root fiber arises from the rostral arm of the loop, while there can be no doubt that the caudal arm of the loop extends on caudad into the tract. The appearance of the fiber at the branching would indicate that the fiber from which this root collateral arises is a descending process of a tract neurone. This is the case also in figure 22, which is drawn from the level of the ninth myotome of an embryo of the same stage of development. The arching outward of the tract fibers at the origin of the root is apparent here also, and particularly is this true in figure 23, which is drawn from a different focal plane of the same field. The disappearance of the more mesial fibers of the tract in the middle of the loop as shown in figure 23 is caused by their passing out of the plane of section as they deflect from their regular longitudinal course in the tract. This deflection in the fibers of figure 23 is directly towards the origin of the root in figure 22 . In the case of figure 21, which is drawn from the root of the eighth myotome of the same embryo as the last, the root collateral has the appearance of arising from an ascending process. The plane of section, however, is here very oblique and the exact relations difficult of interpretation. The root fiber arises from a tract fiber which appears to be the axone of the cell marked $V C$, and the direction of this main process is almost if not quite laterad. The relations here are probably the same as those demonstrated by a different method in figures 12 and 13 .

The most advanced stage of development of the ventral root system under consideration is represented in figure 28 , which is drawn from the first ventral root of an embryo of the early swimming stage. In this case a densely impregnated fiber which lies deeply embedded in the ventro-lateral tract arches outward slightly towards the root of the first spinal nerve and gives off a collateral $(V F)$ which passes directly laterad among the fibers of the tract and enters the nerve root. 


\section{DISCUSSION OF THE RELATIONS IN GENERAL}

From the foregoing particular descriptions it is obvious that neurones of the somatic motor column become well differentiated and typically oriented in the spinal cord before the ventral roots appear in the corresponding level. The earliest demonstrable root fibers arise as collaterals from these neurones. The great majority of clear cases indicate that the collaterals regularly arise from descending processes excepting in the most rostral nerves. In these nerves - the first, second and possibly the third pairthe root collaterals may arise from ascending processes.

This origin of the motor roots from descending processes of tract neurones is in harmony with the general process of integration of the nervous system, which, in these early periods of development, conducts, in the interest of locomotion, all stimuli from the skin directly to the rostral end of the muscle system, and thence caudad so as to produce a wave of contraction that progresses cephalo-caudad. Since the neurones of the descending tract are at the same time the neurones of the ventral roots their polarization would require that root fibers arise from the neurite, or descending process. In the region of the most rostral myotomes, however, the neurites seem to ascend in the motor column. While this may seem at first thought to be inconsistent with the general plan of integration of the motor system it is not so in reality, for, while it is necessary for locomotion that the wave of contraction be stimulated in cephalo-caudal progression, the simultaneous contraction of several of the most rostral myotomes of the same side would not interfere with the efficiency of the movement. Such simultaneous contraction of myotomes in the rostral region would tend to be produced by the introduction of ascending processes in the motor path to the myotomes, for, in embryos of the early flexure stage, the sensory field lies chiefly caudad, in the territory of the giant ganglion cells, and the sensory path ascends to the region of the most rostral myotomes before it can reach the motor system. This it accomplishes at the level of the most rostral myotomes and from this center the motor column can participate in the ascending conduction to 
the most rostral one or two, or possibly three myotomes without interfering with the locomotor efficiency of the muscular system.

In later stages of development the more rostral ventral roots certainly receive collaterals also from descending processes of the tract. This is correlated with the introduction of the cranial sensory field through the descending trigeminal tract, the ventral commissure and the extension rostrad of the motor column.

It seems necessary, therefore, to regard the motor column at the level of the most rostral myotomes to be both descending and ascending in embryos of the early swimming stage. Just how far caudad this ascending conduction may occur in the column it is impossible to say at present. There are suggestions that it advances caudad in development pari passu with the extension caudad of the ventral commissure, but this is not demonstrable with the material at hand. A more critical discussion of this question must await the description of the sensory and commissural systems.

In the very early condition the axone of the tract bends outward slightly at the origin of the root collateral (figs. 2, 4, 9, 11). Later the outward deflection at this point affects numbers of fibers and a large element of the tract bends outward in an abrupt loop, from or near the tip of which the root collaterals arise (figs. 22, 23, 24, 25, 27). This arrangement suggests Johnston's figure of the dorsal roots of Amphioxus ${ }^{2}$ where fibers of the tract deflect out in long loops into the dorsal roots. Johnston suggests that this condition is produced by the outward migration of cells from the cord along the roots. No such agency, however, can account for the condition in Amblystoma embryos for during the periods under consideration there is no perceptible migration of cells from the cord into or along the roots. Furthermore, to recur to the condition in Amphioxus, Johnston describes the viscero-motor fibers as running some distance in the cord longitudinally before they go out into the root. The idea that some at least of these deflected fibers in Amphioxus may be motor immediately suggests itself. Those long loops into the root may

J. B. Johnston, The cranial and spinal ganglia and the viscero-motor roots in Amphioxus; Biological Bulletin, vol. 9, no. 2, fig. 4. 
be concerned in the origin of root fibers in Amphioxus as they certainly are in Amblystoma.

The further development of the definitive ventral roots probably involves the differentiation of neurones within this primary somatic motor column. The primary condition consists in a slender collateral which arises from a comparatively large axone that has been oriented in the column for some time before the collateral makes its appearance (figs. 14, 20, 7, 9, 4). Neurones entering the root later progressively enlarge their collateral branch at the expense of the main process to the tract (figs. 10, 11, 12, 13). This change probably involves the differentiation of neurones in the immediate vicinity of the primary root after the collateral has established itself. As the muscular function of the myotome increases neurones arise which devote their entire neurite to the formation of the root while their dendrites have only insignificant or a very subordinate part in the longitudinal conduction. Such a mode of later differentiation is not established in detail but numerous observations render it plausible.

\section{THEORETICAL CONSIDERATIONS}

\section{Regulation in the origin of the motor roots}

There is a definite correlation of the outgrowth of the primary root collateral and differentiation within the myotome. The collateral always grows to the middle of the muscle cell and applies itself to the cell at the point opposite a large nucleus against which impinges a conspicuous mass of pigment. In the earlier condition of the mesoderm pigment is quite generally distributed as small granules throughout the cells of the myotome. As the cells differentiate into muscle cells this pigment becomes segregated into this central mass. This is evidence of some sort of a polarization of the middle of the muscle cell with reference to the ends. The motor fiber invariably grows to this central region of polarization. The exact time relations between the outgrowth of the collateral and the centralization of pigment are not yet determined but the two processes are very distinctly correlated. 
It is further obvious that the differentiation of the neurones of the motor column and their orientation in the latero-ventral tract are correlated with the more general differentiation of the mesoderm into myotomes. It seems plausible, therefore, that the general and more diffuse process of differentiation within the mesoderm stimulates the differentiation of the motor neurones and their orientation and outgrowth into the tract in a longitudinal direction, while the more localized differentiation within the myotome, related directly to muscular activity, stimulates the origin of the collateral, its growth laterad through the limiting membrane of the cord and its advance to the muscle cell. This hypothesis is founded only on the general growth processes. It is susceptible to experimentation by growth of the tissues in vitro and it is hoped that this method may yet be applied to the details of this problem of correlative development.

\section{Questions of cytomorphic and functional development}

It has been noted above that ventral root fibers occur in their full relation between the spinal cord and the muscle some time before the muscles can be stimulated through the sensory field. In one embryo of this stage in development as many as twelve pairs of roots exist. It is clear, therefore, that the physiological properties of these root neurones can in no exact sense be determined through stimulation of the sensory fieid, for they may be actually functional for some time before they come under the influence of the sensory nerves. It is only after all the elements of a reflex arc are established that a reaction can be elicited through it. My observations upon these embryos show that all the elements in the primary and most elementary reflex arc do not become established simultaneously. Both the motor and sensory elements in this arc are extensively differentiated and in their usual relation to their end organs for some time before the development of associative neurones puts them into such relation to each other as to make reaction to stimulation possible. It would be illegitimate, therefore, to infer that any particular feature in cytomorphic development within the motor column was 
definitely correlated with the development of nervous function simply because that feature arose simultaneously with the first reactions to stimulation of the sensory field. In the attempt to correlate cytomorphosis with function one should first be sure of the physiological value of the particular nerve center with which he is concerned. Certainty on this point can be established only by intensive studies of systems of neurones in their relation to each other and in relation to the development of particular bodily activity; and the observations presented here, it is hoped, may contribute something towards that end.

\section{The primitive nature of the primary motor column}

The discovery that the primary ventral root fibers are collaterals of neurones of a longitudinal tract places the motor system at once in the same class with the primary sensory system of the aquatic vertebrates, namely, the giant ganglion cell system of fishes and Amphibia. As is well known the primary sensory fibers in these forms are dendritic branches of central cells. This sensory system is generally conceded to be primitive and, since the primary motor system is identical with it in the general plan of organization, this motor system must also represent a primitive condition in the nervous system. While the giant ganglion cell system is known to be transitory in the life history of the individual it is impossible to say whether the primitive condition of the motor system becomes obliterated or simply obscured by the later development of the definitive ventral roots.

\section{The theory of nerve components}

This analysis of the primary motor column and the demonstration of its primitive nature afford new and positive conformation of the central idea of the American theory of nerve components, namely, that the primary and most fundamental divisions of the vertebrate nervous system are longitudinal and differentiated upon the basis of unity of function within each division. These divisions are regarded as somatic sensory, visceral sensory, 
visceral motor and somatic motor. The latter division, in its primary and primitive condition is now found to be, not a 'pile' of discrete metameric divisions more or less indefinitely associated by means of longitudinal connectives, but a 'column' in the strict sense of a continuous, functional unit. The metameric arrangement of the motor roots, as suggested above, is probably determined by regulation and accordingly is not paralleled by a metameric arrangement of neurones within the column. The neuromeric organization of the spinal cord, therefore, in so far as it exists, is a secondary acquisition. 


\section{ABBREVIATIONS}

$A P$, ascending process of a neurone $C$, notochord

$C C$, central canal of the spinal cord

$D C$, giant ganglion cell of the dorsal column

$D P$, descending process of a neurone

$D T$, the tract of the giant ganglion cells, dorso-lateral tract

$M$, myotome
$M C$, muscle cell

$P$, pigment

$S$, spinal cord

$S G$, anlage of the spinal ganglion

$V C$, cell of motor column

$V F$, ventral root fiber

$V T$, ventro-lateral tract of the somatic motor column

$Y$, yolk globule

All the figures have been drawn with the aid of the camera lucida. In all but figures 6 and 8 , with which lower magnification was required, the lenses employed with the camera were the Zeiss Homog. oil-immersion $2 \mathrm{~mm}$. objective and Compensating ocular 6 . In nearly every case the compensating ocular 12 was introduced for eritical study in the completion of the drawings. The numbers introduced in parenthesis in the explanation of particular figures record the serial number of the specimen in the collection, the number of the slide and section. In all figures from longitudinal sections the upper end of the drawing as it is arranged on the plate is directed rostrad in the animal.

Fig. 1 From a specimen $(545,1-6-8)$ which reacted to electrical but not to tactile stimulation; fixation, Van Gehuchten's fluid (alcohol-chloroform-acetic acid); stain, erythrosin and toluidin blue; sections, $5 \mu$, in the frontal plane.

Fig. 2 From a specimen $(546,6-3-2)$ of the same description and treated with the same methods as the last.

Fig. 3 From a specimen $(476,1-2-20)$ of the early flexure stage; fixation, sublimate-acetic; staining, alum carmine in toto and Lyon's blue in 95 per cent alcohol; sections, $10 \mu$, in the frontal plane.

Fig. 4 From the same specimen $(476,1-2-16)$ as the Iast.

Figs. 5 to 6 From a specimen $(475,2-1-8)$ of the same description and treatment as the last.

Figs. 7 to 8 From the same specimen $(475,1-3-12)$ as the last. 

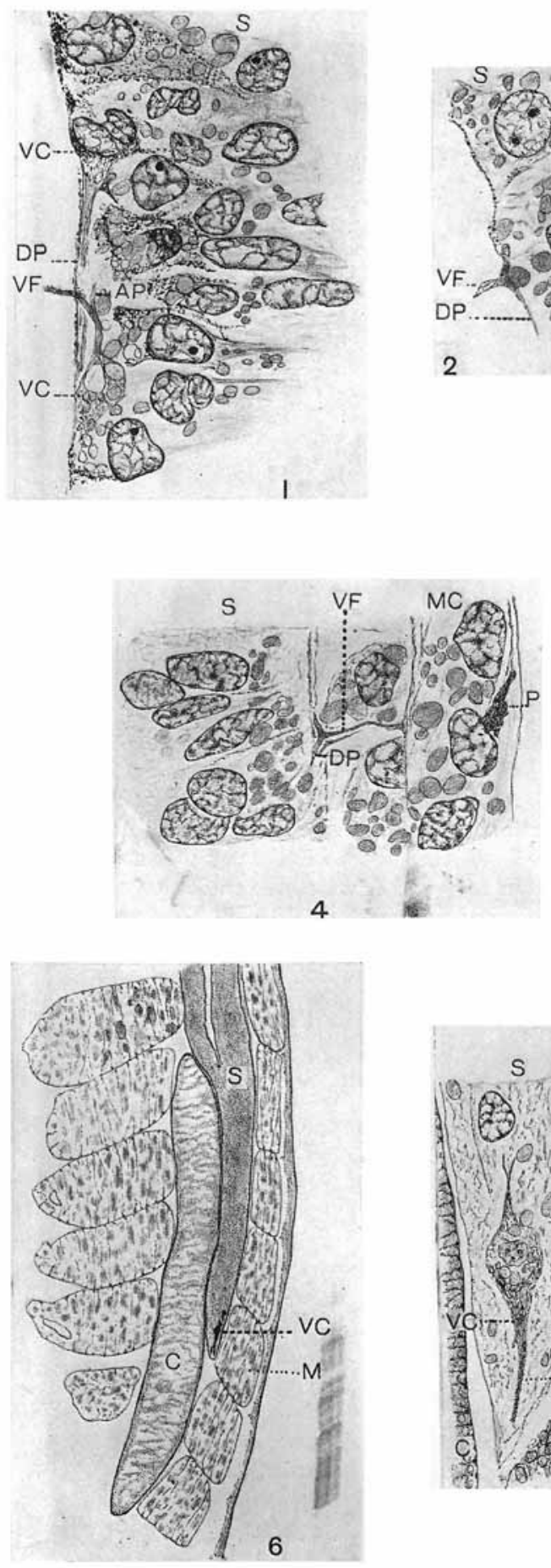
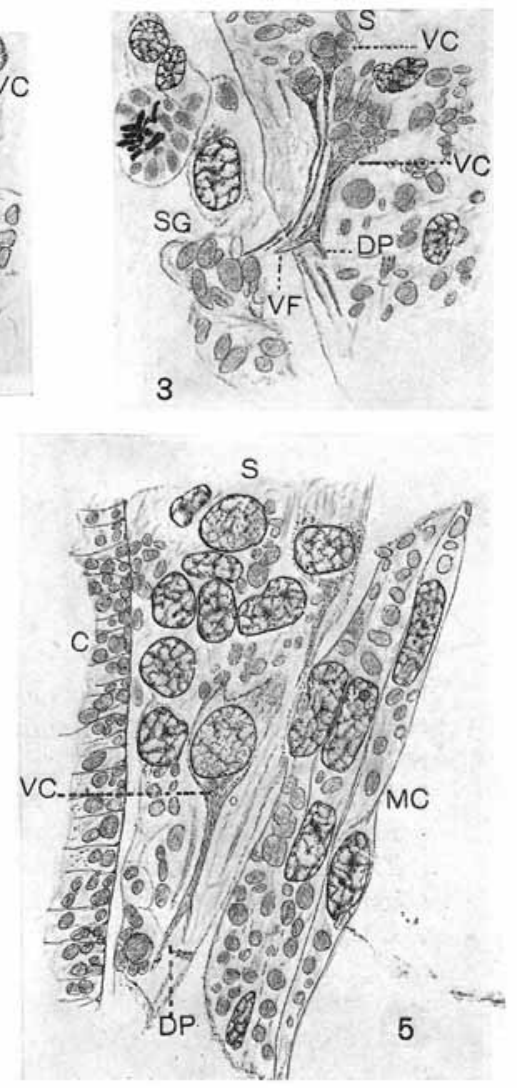

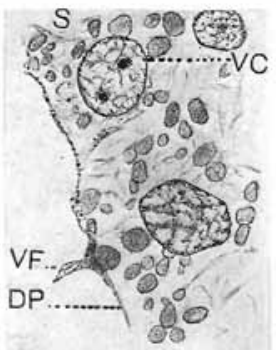

2 
Fig. 9 From a specimen $(551,5-2-16)$ of the early flexure stage; fixation, Van Gehuchten's fluid; staining, erythrosin and toluidin blue; sections, $7 \mu$, Iongitudinal and in an obliquely vertical plane.

Fig. 10 From a specimen (564, 3-2-19) of the early 'coil-reaction' stage; fixation, Zenker's solution; staining, iron hematoxylin; sections, $7 \mu$, in the frontal plane.

Fig. 11 From the same specimen (564, 3-2-17) as the last.

Figs. 12 to 13 From the same specimen (564, 3-2-18) as the last.

Figs. 14 to 15 From a specimen (555) of the typical 'coil-reaction' stage; fixation, Van Gehuchten's fluid, staining, erythrosin and toluidin blue; sections, $7 \mu$, longitudinal and in an obliquely vertical plane.

Fig. 16 From a specimen (505, 1-2-15) of the 'S-reaction' stage; fixation, sublimate-acetic; staining, alum carmine in toto and Lyon's blue in 95 per cent alcohol acidulated slightly with hydrochloric acid; sections, $10 \mu$, in the frontal plane.

Fig. 17 From a specimen (503, 1-2-11) of the 'S-reaction' stage, treated as the last but in the sagittal plane. 

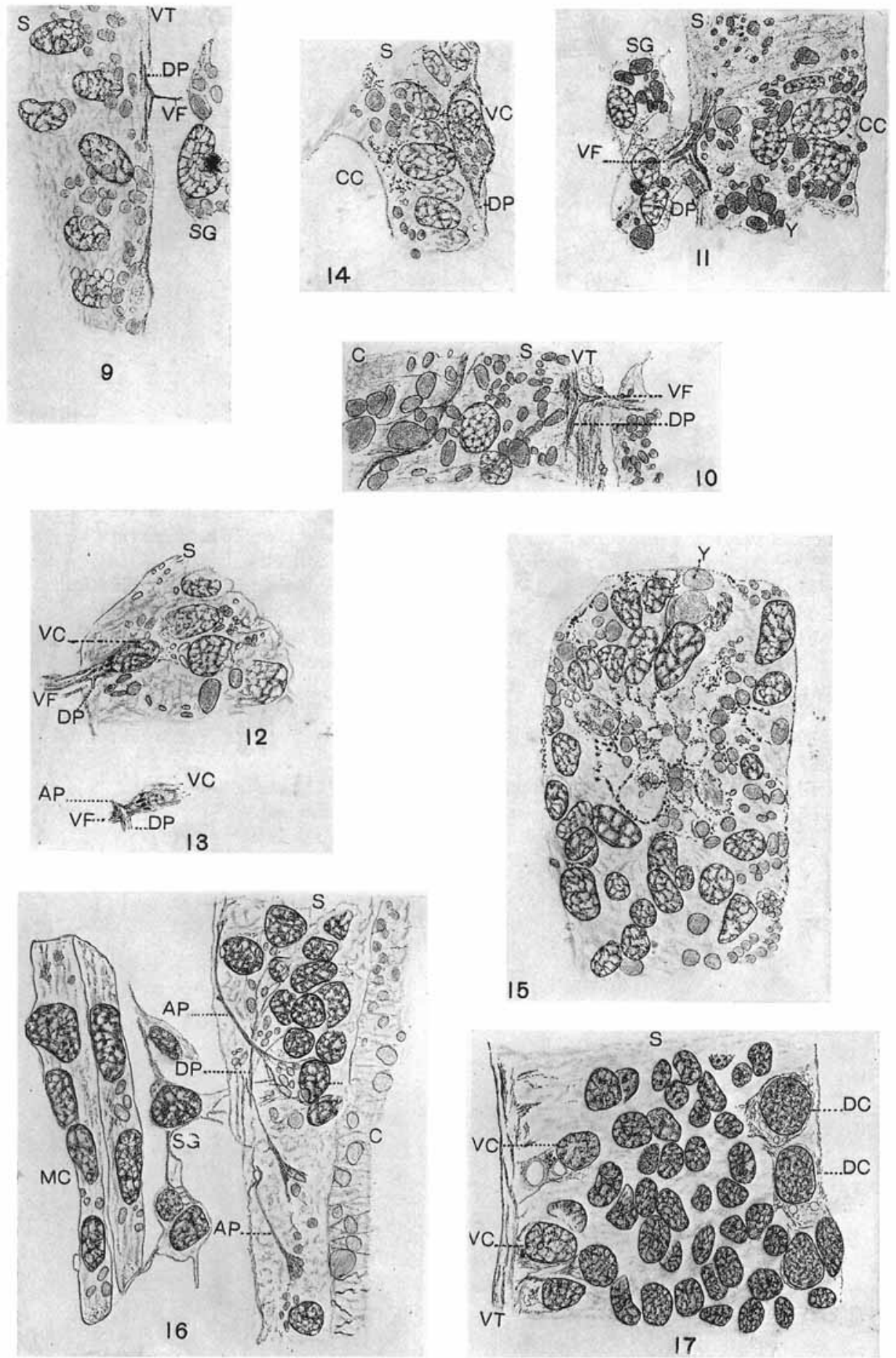
Fig. 18 From a specimen (444, 4-4-6) of the early swimming stage; fixation, sublimate acetic; staining, Bomer's hematoxylin and orange $G$ in 60 per cent alcohol slightly acidulated with hydrochloric acid; sections, $10 \mu$, transverse.

Fig. 19 From the same specimen $(444,2-2-5)$ as the last.

Fig. 20 From a specimen $(570,2-2-5)$ of the early swimming stage; fixation, Zenker's fluid; staining, iron hematoxylin; sections, $7 \mu$, in the frontal plane.

Fig. 21 From a specimen $(412,5-1-3)$ of the early swimming stage; fixation and impregnation according to Paton's modification of the nitrate of silver method; sections, $6 \frac{2}{3} \mu$, in the frontal plane.

Figs. 22 to 23 From the same specimen $(412,5-2-3)$ as the last.

Fig. 24 From a specimen $(408,4-3-9)$ of the early swimming stage; method and sectioning the same as the last.

Figs. 25 to 26 From the same specimen $(408,4-3-10)$ as the last.

Fig. 27 From the same specimen $(408,4-3-11)$ as the last.

Fig. 28 From the same speeimen $(408,3-2-7)$ as the last. 

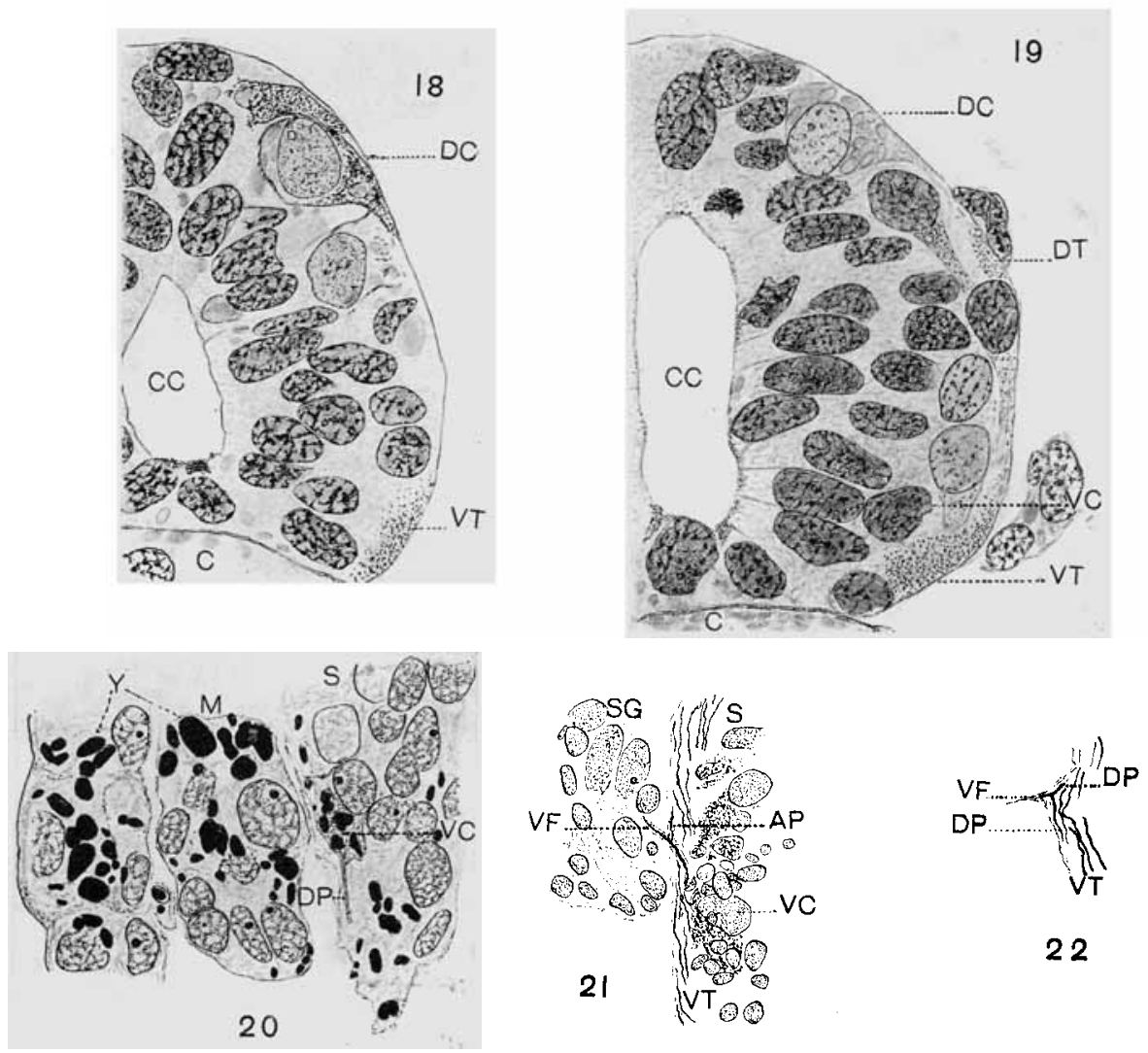

22

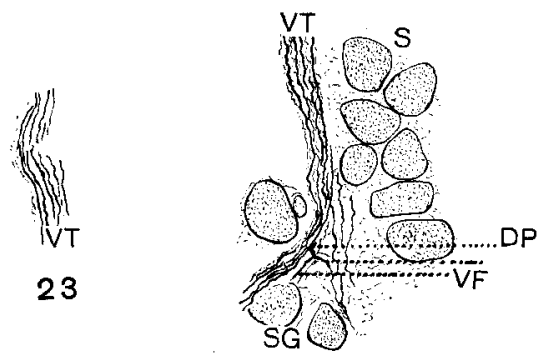

24

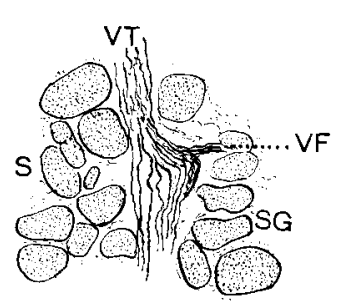

$\underset{D P}{V F} 26$

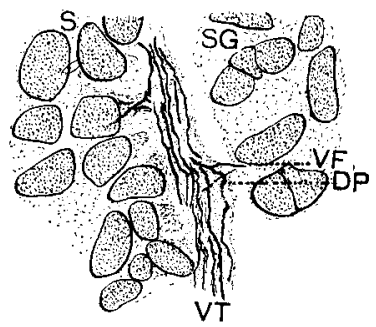

27

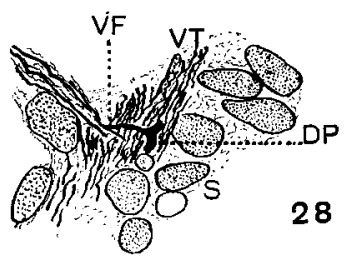

